\title{
The Effect of Various Hormones on the Humoral Immune Response of Murine Lymphocytes in Vitro
}

\author{
Kunihiro Okamura, Masaki Kuramoto, Yohichi \\ Hamazaki and Masakuni Suzuki \\ Department of Obstetrics and Gynecology, Tohoku University \\ School of Medicine, Sendai 980
}

\begin{abstract}
Okamura, K., Kuramoto, M., Hamazaki, Y. and Suzuki, M. The Effect of Various Hormones on the Humoral Immune Response of Murine Lymphocytes in Vitro. Tohoku J. exp. Med., 1978, 126 (3), 235-238- Human chorionic gonadotropin (HCG) and steroid hormones were tested for their direct effects on murine lymphocyte humoral response to sheep RBCs using an in vitro culture system. A significant increase in plaque forming cells (PFC) was induced by the addition of HCG at a dose of either $50 \mathrm{i} . u$. or $500 \mathrm{i} . \mathrm{u}$. However, no increase was observed by the addition of steroid hormones. HCG may contribute to the success of fetal allografts by increasing the number of blocking antibodies in the early stages of pregnancy. —_ hormones; humoral immune response
\end{abstract}

The mechanism by which an antigenically foreign fetus can avoid immunological attacks by the maternal immune reaction remains to be elucidated.

A number of reports have shown that the depression of maternal immunity seems to be important. This situation points to the possibility that the immunosuppressive factors in late pregnant serum may lead to the success of fetal allografts.

Previously, we attempted to purify the immunosuppressive factors in pregnant serum and found that alpha-globulins might be the main component of these factors (1977). We have also been interested in the lymphocyte reaction during pregnancy. In this paper we report on the direct effect of various hormones on the murine lymphocyte response to sheep RBCs in an in vitro culture system.

\section{Methods}

Twelve to 14-week-old, nulliparous ddYF mice were used throughout the experiments. The in vitro immunization of murine lymphocytes was applied according to the method described by Kamo et al. (1976). In brief, five to ten million splenocytes were cultured in $2.5 \mathrm{ml}$ of RPMI 1640 medium supplemented with $10 \%$ fetal calf serum and $2 \times 10^{6}$ sheep RBCs in a flat bottom vial $(45 \times 14 \mathrm{~mm}$, A.H. Thomas Co.) covered with a Morton steel closure. $0.5 \mathrm{ml}$ of "nutrient cocktail" mixture was added to the culture, which was then maintained at $37^{\circ} \mathrm{C}$ in a humidified atmosphere of $5 \% \mathrm{CO}_{2}$ and $95 \%$ air. Five days after culture initiation, the number of direct hemolytic plaque forming cells (PFCs) was evaluated according to the method of Cunningham. Three different hormones, human chorionic gonadotropin (HCG, Mochida Pharmaceutical Co., Ltd.) as gonadotropin, $11_{\alpha-}$ succinoyloxy-progesterone as progesterone, and estradiol phosphate as estrogen were tested.

Received for publication, February 6, 1978. 
Each hormone was dissolved at various concentrations in $0.1 \mathrm{ml}$ of RPMI 1640 medium and added to the vials at the beginning of culturing.

Human serum albumin (Miles Labs., Inc.) was examined as a heterologous protein to murine lymphocytes in the same manner as for HCG.

\section{RESULTS}

As can be seen in Fig. 1, a significant increase in the PFC count was observed in each culture to which was added either 50 international unit (i.u.) or 500 i.u. of HCG. Low doses of HCG did not influence the PFC count. In order to determine whether the effect of HCG might be attributed to the heterologous nature of the murine lymphocytes, tests were carried out using human serum albumin. However, no change in the PFC count was observed by the addition of human serum albumin.

Neither progesterone nor estrogen had any effect on the PFC counts in our system (Fig. 2).

\section{Discussion}

Fabris and Piantanelli (1976) observed that daily administrations of HCG to female mice enhanced the direct PFC count to sheep RBCs and depressed the contact allergic reaction induced by painting the skin of the mouse with picryl

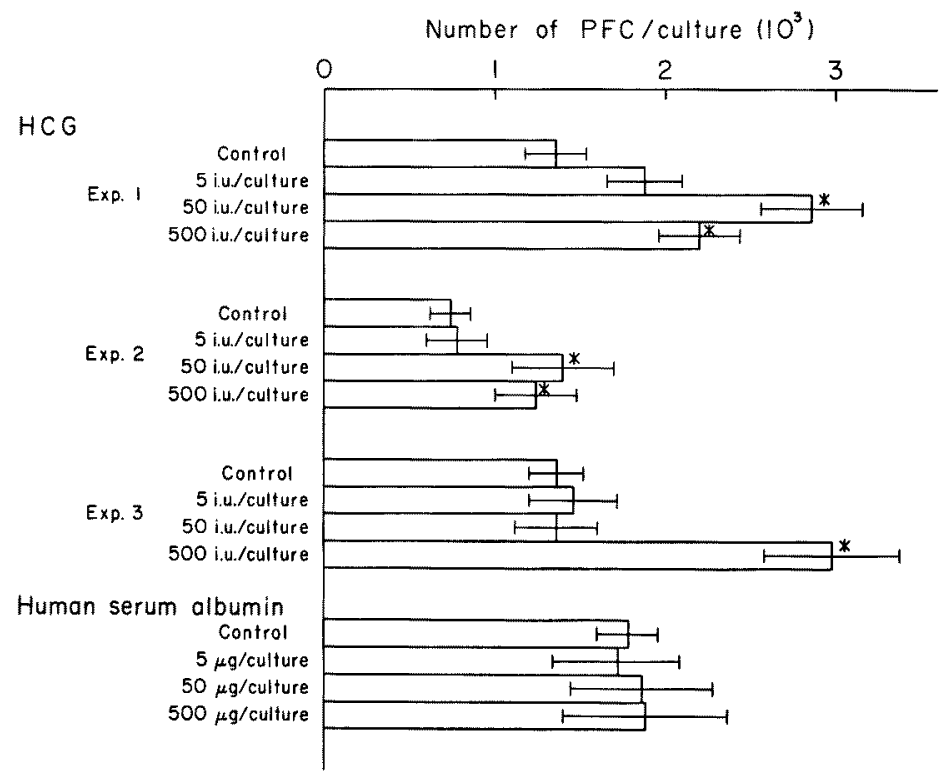

Fig. 1. The effect of HCG and human serum albumin on the in vitro immune response of murine splencotyes.

HCG and human serum albumin were dissolved in $0.1 \mathrm{ml}$ of RPMI 1604 medium and added at the initiation of culturing. All tests were run in triplicate in the determination of PFC and each column expresses the mean value of PFCs \pm s.D.

* $p<0.05$. 


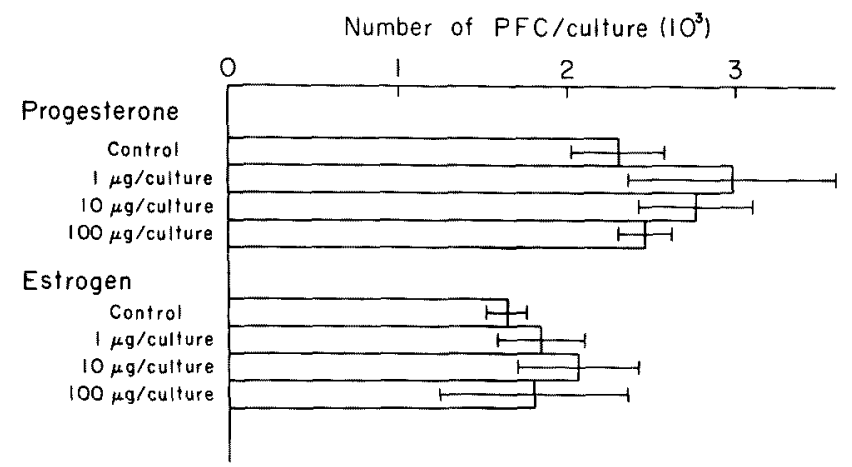

Fig. 2. The lack of influence of steroid hormones on the vitro immune response of murine splenocytes.

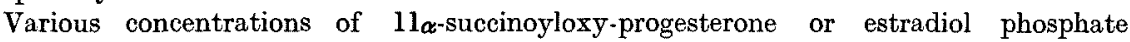
dissolved in $0.1 \mathrm{ml}$ of RPMI 1640 medium were added at the initiation of culturing.

chloride. Moreover, he reported that not only the enhancement of humoral immunity but also the depression of cell-mediated immunity occurred in pregnant mice. The present paper indicated that the addition of $\mathrm{HCG}$ enhanced the count of $\mathrm{PFC}$ in the in vitro systems. It does not seem likely that the heterologous nature of $\mathrm{HCG}$ to murine lymphocytes is responsible to this enhancement of the PFC, since human serum albumin did not influence this count in our system.

Strelkauskas et al. (1975) showed that the ratio of human T to B cells was inverted in early pregnancy. This increase in the $\mathrm{B}$ cell level may parallel the HCG rise at this period. Considering our results and other observations it can be said that HCG could react directly with the lymphocytes and produce the increase in the $B$ cell levels. However, it has not been ascertained whether HCG controls the lymphocyte which regulates the $B$ cell functions.

Hellström and Hellström (1974) proposed the presence of blocking antibodies in malignant tumors. This system might also exist during early pregnancy when a similar type of antibody may increase in number under the existence of HCG. Probably HCG may help the acceptance of fetal allografts by this mechanism.

HCG has been reported to depress cell-mediated immunity (Kaye et al. 1971). However, it remains controversial whether purified HCG could produce suppression of lymphocyte blastogenesis (Caldwell et al. 1975; Beck et al. 1977).

It is quite possible that $\mathrm{HCG}$, secreted in large amounts during early pregnancy, could play an immunological role in the acceptance of fetal allografts.

Steroid hormones have been verified for their suppressive action to lymphocyte blastformation (Wyle and Kent 1977). But, alterations in the PFC counts were not observed by the addition of these hormones in our system.

\section{References}

1) Beck, D., Ginsburg, H. \& Naot, Y. (1977) The modulating effect of human chorionic gonadotropin on lymphocyte blastogenesis. Amer. J. Obstet. Gynecol., 129, 14-20. 
2) Caldwell, J.L., Stites, D.P. \& Fudenberg, H.H. (1975) Human chorionic gonadotropin: Effects of crude and purified preparations on lymphocytes responses to phytohamagglutinin and allogenic stimulation. $J$. Immunol., 115, 1249-1253.

3) Fabris, N. \& Piantanelli, L. (1976) Effect of chorionic gonadotropins on humoral and cell mediated immunity. Adv. exp. Med. Biol., 66, 635-638.

4) Hellström, K.E. \& Hellström, I. (1974) Lymphocyte-mediated cytotoxicity and blocking serum activity to tumor antigens. Advanc. Immunol., 18, 209-277.

5) Kamo, I., Pan, S.H. \& Friedman, H. (1976) A simplified procedure for in vitro immunization of dispersed spleen cell cultures. J. Immun. Meth., 11, 55-62.

6) Kaye, M.D. \& Jones, W.R. (1971) Effect of HCG on in vitro lymphocyte transformation. Amer. J. Obstet. Gynecol., 109. 1029-1030.

7) Okamura, K., Kuramoto, M., Hamazaki, Y. \& Suzuki, M. (1977) Partial purification of an immunosuppressive factor in pregnant serum. Proceedings of the 29th annual scientific meeting. Acta obst. gynaec., 29, 1895. (Japanese)

8) Strelkauskas, A.J., Wilson, B.S., Dray, S. \& Dodson, M. (1975) Inversion of levels of human $\mathrm{T}$ and $\mathrm{B}$ cells in early pregnancy. Nature, 258, 331-332.

9) Wyle, F.A. \& Kent, J.R. (1977) Immunosuppression by sex steroid hormones. Clin. exp. Immunol., 27, 407-415. 